\title{
Validity and reliability of a radio positioning system for tracking athletes in indoor and outdoor team sports
}

\author{
Thuraiappah Sathyan - Richard Shuttleworth • \\ Mark Hedley $•$ Keith Davids
}

Published online: 5 April 2012

(C) Psychonomic Society, Inc. (outside the USA) 2012

\begin{abstract}
Radio-frequency local positioning systems (LPS) have the potential to provide accurate location information about sport players for performance analysis, making available for study the emergent nature of interpersonal coordination and collective decision-making behaviour among players in both indoor and outdoor sports. However, no available publications have validated the performance of LPS for indoor sports. The objective of this study was to validate the performance of an LPS in an indoor venue and to compare it to performance observed in an outdoor venue using static and dynamic measurements. Our results showed that the absolute positioning errors obtained from the static measurements of the LPS were comparable for both indoor and outdoor venues, with mean errors of $12.1 \mathrm{~cm}$ outdoors and $11.9 \mathrm{~cm}$ indoors. From the dynamic measurements, we analysed the relative position error and the distance estimation performance of the system. The 90th-percentile relative position errors were $28 \mathrm{~cm}$ for the indoor venue versus $18 \mathrm{~cm}$ for the outdoor venue. On average, the LPS overestimated the distance travelled, and the performance was similar for both indoor and outdoor venues. On a linear course, the mean errors of the distance estimates were $2.2 \%$ of the total distance indoors and $1.3 \%$ outdoors, and
\end{abstract}

T. Sathyan $(\varangle) \cdot$ M. Hedley

Commonwealth Scientific and Industrial Research Organisation, P.O. Box 76, Epping, New South Wales 1710, Australia

e-mail: tsathyan@ieee.org

R. Shuttleworth

Australian Institute of Sport,

Canberra, Australian Capital Territory, Australia

K. Davids

Queensland University of Technology,

Brisbane, Queensland, Australia on a nonlinear course, they were $2.7 \%$ indoors and $3.2 \%$ outdoors.

Keywords Athlete performance analysis · Location tracking $\cdot$ Radio-frequency positioning $\cdot$ Local positioning system

Accurate tracking of the location and movement of athletes during training and competitive performance provides a basis for improved performance analysis, leading to the enhancement of tactical behaviours and the development of new training strategies. It may also assist in improving understanding of the learning process by enabling sport scientists to investigate how dynamic interpersonal interactions emerge under varying practice constraints in team games (Araújo, Davids, \& Hristovski, 2006; Davids, Button, \& Bennett, 2008; Schmidt, Fitzpatrick, Caron, \& Mergeche, 2011). Furthermore, insights may be gained through location tracking that can help address some of the concerns highlighted by such academics as Glazier (2010) and Vilar, Araújo, Davids, and Button (2012) about current and future directions in sport performance analysis.

From an ecological-psychology and dynamical-systemstheory approach to examining complex systems behaviour in sport (Araújo, Davids, Chow, \& Passos, 2009; Araújo, Davids, \& Serpa, 2005; Hristovski, Davids, \& Araújo, 2009; McGarry, Anderson, Wallace, Hughes, \& Franks, 2002; Renshaw, Davids, Chow, \& Shuttleworth, 2009), a growing body of research has aimed to further our understanding of the processes underlying perceptual motor tasks using appropriate methodologies that will allow researchers to analyse the complexity of dynamical processes in sport performances. The challenge for sport scientists currently 
investigating multi-agent behaviour in team environments is that they require accurate and reliable measurement of system and (sub)system dynamics taken in situ in order to observe emergent interactions between players. More importantly, this advancement in technology precision may afford experimental psychologists and movement scientists the opportunity to critically discover simple measures and key parameters over different time scales of performance that will capture the complexity of the system.

The main location tracking technologies currently used in sport performance analysis are either GPS- or video-based, and a number of studies have investigated the validity and reliability of these systems (Duthie, Pyne, \& Hooper, 2003; Gray, Jenkins, Andrews, Taaffe, \& Glover, 2010). While these studies have shown that GPS- and video-based tracking techniques are sufficiently accurate, their limitations have also been demonstrated: Their accuracy was significantly impacted in short sprints, high-intensity runs, and agile movements with rapid changes in directions. Furthermore, GPS does not operate indoors, and therefore cannot be used to study performance and training in indoor sports. The commercial videobased tracking systems also require a degree of manual intervention, for example, to robustly identify and label players in order to obtain positional data (Barris \& Button, 2008).

Local positioning systems (LPS) are an alternative technology that uses radio-frequency signals to measure the range between mobile nodes carried by the players and a number of infrastructure nodes (referred to as anchor nodes) at known locations. The range measurements are used to provide highly accurate estimates of the location of each athlete being tracked. The operating principle of the LPS is similar to that of GPS; however, since LPS uses a locally deployed infrastructure rather than the remote, satellitebased infrastructure used by GPS, it can be used for indoor sports as well.

The study by Frencken, Lemmink, and Delleman (2010) validated an LPS in soccer-specific conditions and concluded that it is a valid tool for recording the positions of players during team games and practices. To the best of our knowledge, however, no study exists that validates the performance of LPS for indoor sports. The aim of this study was thus to validate a radio-frequency LPS for indoor sports and to compare the performance to its performance for outdoor sport activity using both linear and nonlinear methods of data analysis. The system that we consider-called the Wireless Ad hoc System for Positioning (WASP)—was developed by the Commonwealth Scientific and Industrial Research Organisation. The system operates in the $5.8-\mathrm{GHz}$ ISM (industrial, scientific, and medical) band and uses 125 $\mathrm{MHz}$ bandwidth. WASP allows both anchor and mobile nodes to transmit in preallocated time slots of 2.5-ms duration using a time division multiple access protocol to provide reliable and periodic location updates. The system is typically configured to use 40 slots consisting of 12 anchor nodes and 27 mobile nodes, each using one slot. The last slot is kept free for control signalling. This provides an update rate of $10 \mathrm{~Hz}$ for each mobile node. The system can be configured to provide a higher update rate for a smaller number of mobile nodes, with a maximum locate rate of $200 \mathrm{~Hz}$ when a single player is tracked. The mobile nodes carried by the players measure $90 \times 50 \times 25 \mathrm{~mm}$ and weigh $100 \mathrm{~g}$. Further technical details of the system, including the hardware architecture, protocols, and signal processing algorithms, can be found in Sathyan, Humphrey, and Hedley (2011) and the references cited therein.

\section{Method}

Ten volunteers each participated in both the indoor and outdoor performance analysis trials. The participants comprised a mix of elite-level athletes from the Australian Institute of Sport's basketball, netball, rugby, and soccer programmes, including six males (age: $18 \pm 1.4$ years) and four females (age: $19 \pm 1.2$ years). The physiological characteristics of the participants (weight, height, fitness level), although they varied, were not recorded since they were deemed not to affect the localisation process. The procedure was explained to the participants before the start of the experiment, and 2 participants were involved in the data collection procedure at any given time. The instructions to participants were kept to a minimum and remained the same for both the indoor and outdoor testing protocols. Participants $(n=10)$ were asked to complete one trial for each condition $(n=4)$ - (1) walk, (2) jog, (3) run, and (4) sprint - with a 3-min break between trials to prevent fatiguing. The same protocol was used for linear and nonlinear tests and for indoor and outdoor trials. The total number of trials for each of the 10 participants in the indoor trials was eight, and likewise for the outdoor trials. Thus, a total of 160 trials were undertaken for validation. It was not deemed important for analysis purposes to control for relative speeds across participants, and therefore their self-preferred speeds were used in the testing protocols for the walk, jog, run, and sprint conditions.

A linear and a nonlinear course (refer to Fig. 1) were marked on the ground using chalk, and the participants were asked to walk, jog, run, and sprint along the two courses after standing still for nearly $15 \mathrm{~s}$. Participants were asked to follow the marked lines as closely as possible. The linear courses had nominal lengths of $28 \mathrm{~m}$ indoors and $30 \mathrm{~m}$ outdoors, and the nonlinear course had a nominal length of $27.6 \mathrm{~m}$ both indoors and outdoors. These course lengths were measured using a tape measure and were crosschecked with the distance calculated from the survey. 


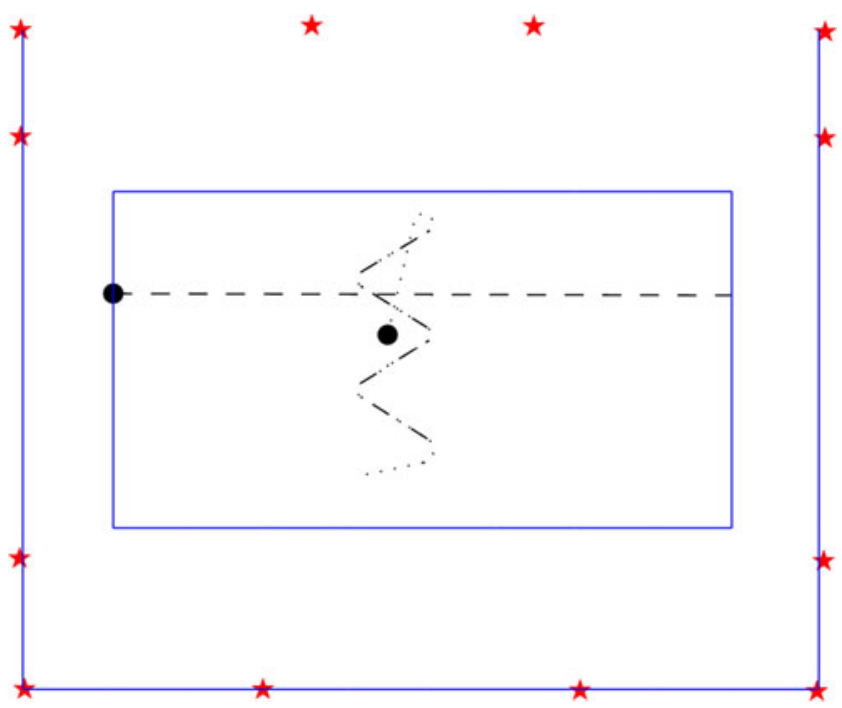

Fig. 1 Anchor layout and the linear (black dashed line) and nonlinear (black dotted line) courses used in the indoor basketball court. Red stars denote the anchor nodes, and black dots denote the starting points. Blue solid lines show the court layout and the walls on which the anchor nodes were mounted

The nonlinear course selected was an agility test course used at the Australian Institute of Sport (AIS). The course indicates an athlete's overall agility and ability to change direction with speed. The test course is currently used by professional clubs in the Australian Football League (AFL) and by various AIS sports programmes to test a player's agility levels. The movement patterns required of the athlete to complete the dynamic performance test included two short straight-line sprints to start and finish, three sharp left-hand corners and two sharp right-hand corners, in alternate order. This effort requires acceleration, deceleration, and changes in velocity in order to successfully navigate the course.

The WASP platform used in the trials is an LPS that provides high-accuracy localisation performance in both indoor and outdoor sports. It is a range-based system that, unlike the LPS considered in Frencken et al. (2010), requires no cabling, making it suitable for rapid deployment across multiple venues for performance analysis tasks.

The indoor trial was conducted on an indoor basketball court, and the outdoor trial was conducted on a rugby field. Twelve WASP nodes were used in both indoor and outdoor trials as anchor nodes whose locations were measured using a conventional survey with an accuracy of a few centimetres. The anchor nodes were distributed approximately uniformly around the playing area, with four anchor nodes each on the longer sides and two each on the shorter sides. The anchor setup used on the basketball court is shown in Fig. 1, and a similar setup was used on the outdoor field. Ten of the anchor nodes used in the indoor trial were mounted on the walls of the indoor venue, and the other two were mounted on tripods. All of the anchor nodes were mounted on wooden poles located around the rugby field.

The walls of the basketball court were made of concrete, and the roof consisted of steel. The environment created significant multipath conditions - that is, the received signal not only consisted of the direct path signal, but also of signals from the reflected paths. The multipath effect must be accounted for when processing the range measurements to obtain the locations of the mobile nodes. The rugby field area was an open field with no buildings near the playing area, and hence, there was no disruption to the operation of the LPS due to multipath conditions.

The WASP platform measured the range between the mobile nodes carried by the participants and the anchor nodes. The measured ranges were then post-processed in a computer to locate the participants in situ with respect to the anchor nodes. The location update rate was set to $10 \mathrm{~Hz}$ for each mobile node.

The participants wore an unobtrusive strap-on vest containing a mobile WASP node, which was positioned in the middle of the upper back. Two other mobile WASP nodes were fixed to a ruler with a known separation of $40 \mathrm{~cm}$, and the ruler was horizontally attached to the upper back of each participant just above the vest node. None of the participants reported any discomfort in carrying the three nodes. During the normal operation of the system, only a single node attached to the player (e.g., the one in the pouch) would be used to locate the player. The two nodes attached to the ruler were used in the trial to obtain the relative positioning accuracy, for validation purposes only.

The locations of the nodes were obtained from the range measurements using a least-squares algorithm, and these noisy locations were processed using a Kalman filter to obtain smoother estimates of the path. Outlier range measurements are inevitable in practical measurement systems, and a validation gating technique was used to identify and remove them. In the coarse validation gating used in our algorithm, when the range measurements between a mobile node and the anchor nodes became available, the predicted location of the mobile node obtained using the Kalman filter was used to find the predicted range to the anchor nodes. If the difference between the predicted range and the corresponding measured range was greater than the predefined threshold of $3 \mathrm{~m}$, the range measurement was deemed an outlier and was removed from further consideration. Further details of the positioning algorithm we used can be found in Hedley, Sathyan, and Mackintosh (2011).

In the LPS used in the study of Frencken et al. (2010), the calculated positions of the mobile nodes are output directly, and the user of the system does not have access to the measured ranges. The WASP platform, however, provides the measured ranges to the user and allows for custom-made location calculation algorithms. This allowed us to study the 
effects of applying filtering on the estimation accuracy of location and distance travelled.

Static and dynamic measurements were collected in both indoor and outdoor settings to analyse the performance of the WASP system. Static measurements were collected by placing 15 WASP nodes in the playing area in both indoor and outdoor venues on a tripod. The locations of the static nodes were surveyed along with those of the anchor nodes. In the static case, the value of the absolute location error was calculated by comparing the surveyed position to that obtained from the system.

In the case of dynamic measurements, since a more accurate system that can provide the actual course the participants take was unavailable, only the evaluation of relative accuracy was possible. The relative position error was calculated by plotting the separation distance between the two mobile nodes attached to the ruler from the estimated location of the two nodes and comparing it to the known true separation distance between the nodes.

The distance travelled by a participant was calculated by summing the displacements between two consecutive estimated locations between the start and end points of the course. The start and end points were obtained from the data by comparing the points in the data to those of the surveyed locations of the start and end points of the course.

\section{Results}

We processed the static measurements collected in the two venues to determine the range error distribution and absolute location error performance of the system. Figure 2 shows the range error distribution in both the indoor and outdoor venues. It can be clearly seen that while the outdoor range error distribution can be well approximated by a Gaussian

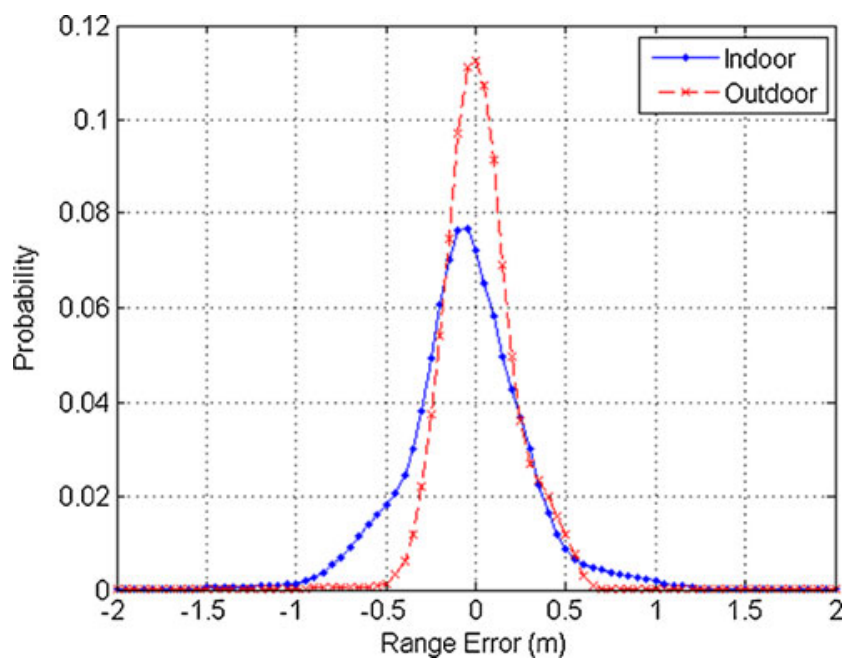

Fig. 2 Range error distributions in the indoor and outdoor venues distribution, the indoor range error distribution is not symmetric, due to the effects of multipath propagation. It can also be seen that the indoor range measurements have a higher variance, and that there are more range measurements with large errors (heavy-tailed distribution), which are both consequences of substantial multipath propagation. These would lead to large tracking errors in indoor venues if they were not correctly considered by the tracking algorithm.

The absolute location error performance of the system averaged across all of the static nodes had similar characteristics in both the indoor and outdoor venues, with the 90thpercentile error in both cases being around $18 \mathrm{~cm}$. The mean $(\mu)$ and standard deviation $(\sigma)$ were found to be $(\mu \pm \sigma) 12.1$ $\pm 5.17 \mathrm{~cm}$ for the outdoor venue and $11.9 \pm 4.85 \mathrm{~cm}$ for the indoor venue.

The dynamic measurements collected from the nodes attached to the players were processed to validate the relative positioning error and distance-travelled estimation performance of the system. Figure 3 displays a sample of the path measured by the LPS of an athlete along the indoor nonlinear path. The data correspond to the node that was inside the strap-on vest in the indoor setting. It can be seen that the estimated path closely follows the actual course. Similar results were observed in other experiments, both indoors and outdoors.

Figure 4 shows the cumulative density function of the relative positioning error, which provides the probability that the positioning error is less than a given value for both linear and nonlinear courses in the indoor and outdoor venues. The distance estimation error performance of the LPS, in terms of the mean, the standard deviation, and the minimum and maximum of the distance-travelled error, are

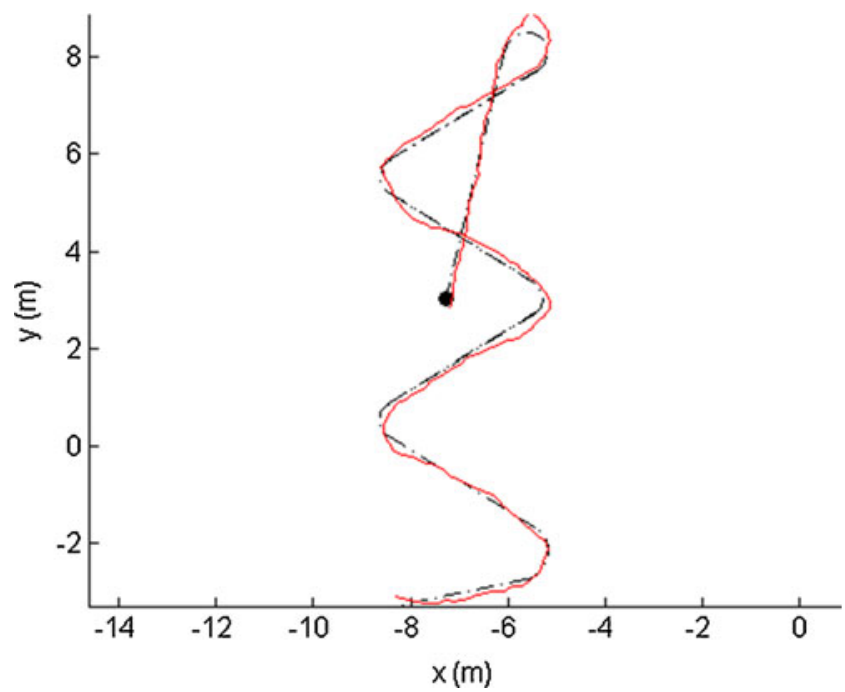

Fig. 3 The nominal trajectory marked on the ground in the indoor basketball court is shown as the black dash-dotted line, and a sample nonlinear trajectory produced by the WASP system is shown as the red solid line 


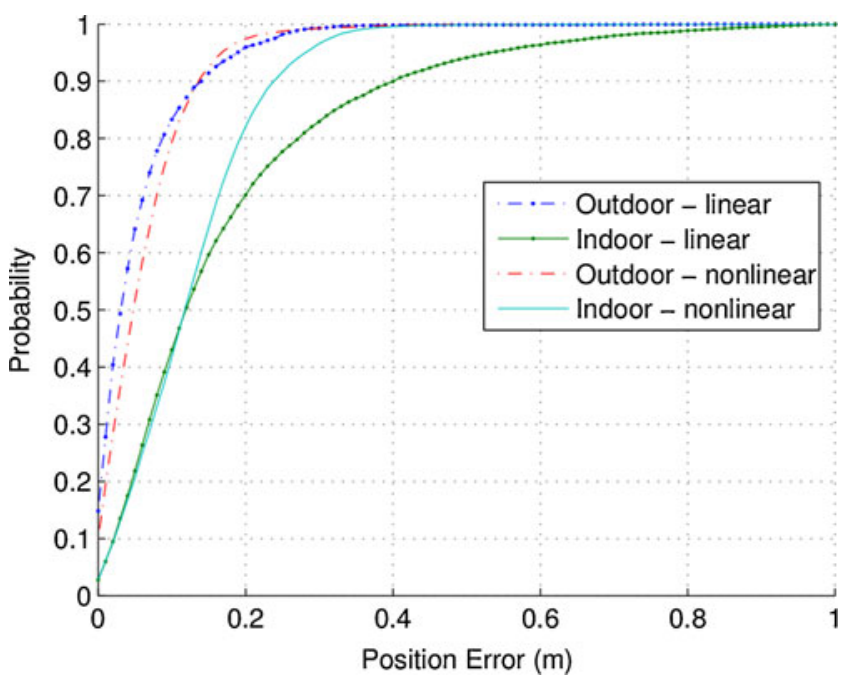

Fig. 4 Relative positioning error performance in both indoor and outdoor venues

presented in Table 1 for the indoor/outdoor linear and nonlinear cases.

The walk and jog trials were merged into a single walk/ jog category, and likewise the run and sprint trials were merged. The statistics in each row of Table 1 were calculated using a total of 20 trials (e.g., the first row of the table is based on the walk and jog results of the 10 participants on the outdoor linear course).

\section{Discussion}

The aim of this study was to validate the effectiveness of the use of an LPS for tracking athletes in indoor sports and to compare the performance with that obtained in outdoor sports. Validation tests were carried out on the basis of the absolute and relative positioning errors and the distance travelled under static conditions and linear and nonlinear motion patterns. Our results demonstrate that the performance of the system is consistent both indoors and outdoors and is sufficiently accurate for tracking athletes.

The absolute position error performance of the system showed good agreement in both environments, with a mean error around $12 \mathrm{~cm}$. The maximum error was less than $30 \mathrm{~cm}$ in both environments. This is the best-case performance that could be expected from the system in both environments, because when nodes are body-worn, the positional accuracy is influenced by body blockage. When the line-of-sight path of the radio-frequency signals between the mobile and anchor nodes is blocked by a player's body, a large error in measured range can occur, which leads to large positional errors. It was reported in Frencken et al. (2010) that the LPS used in that study had an accuracy of around $1 \mathrm{~cm}$ in an outdoor venue for static measurements, which is more accurate than we found in the present study. Unlike in our study, in which the surveyed position was considered as the actual location of a static node, Frencken et al. used the sample mean as the actual location of the static node. Using the sample mean as the actual location, however, masks any bias errors introduced by the system. The static accuracy of the LPS presented by Frencken et al. was also evaluated on the basis of measurements from only three static nodes. Furthermore, the static nodes were confined to a narrow segment of the field, which would mask the effects of variation in the geometric dilution of precision - a measure quantifying the variation in positioning accuracy due to the geometry of the anchor nodes and the mobile node ( $\mathrm{Zhu}$, 1992). We believe that these procedures may have contributed to the improved accuracies observed in the Frencken et
Table 1 Statistics of distance estimate performance for the local positioning system
The statistics in each row were compiled from 2 trials for each of the 10 participants - that is, a total of 20 trials

\begin{tabular}{|c|c|c|c|c|}
\hline \multirow[t]{2}{*}{ Course } & \multirow[t]{2}{*}{ Course Length (m) } & \multicolumn{2}{|c|}{ Estimated Distance } & \multirow{2}{*}{$\begin{array}{l}\text { Mean Error (\%) of } \\
\text { Course Length) }\end{array}$} \\
\hline & & $\mu \pm \sigma(\mathrm{m})$ & $\begin{array}{l}\text { Min and Max } \\
\text { Error }(\mathrm{m})\end{array}$ & \\
\hline \multicolumn{5}{|c|}{ Linear Outdoor } \\
\hline Walk, jog & 30.00 & $30.38 \pm 0.15$ & 0.03 to 0.61 & 1.26 \\
\hline Run, sprint & 30.00 & $30.42 \pm 0.20$ & 0.11 to 0.86 & 1.40 \\
\hline \multicolumn{5}{|c|}{ Linear Indoor } \\
\hline Walk, jog & 28.00 & $28.67 \pm 0.27$ & 0.06 to 1.11 & 2.39 \\
\hline Run, sprint & 28.00 & $28.57 \pm 0.19$ & 0.27 to 0.82 & 2.03 \\
\hline \multicolumn{5}{|c|}{ Nonlinear Outdoor } \\
\hline Walk, jog & 27.60 & $28.44 \pm 0.65$ & -0.55 to 1.76 & 3.04 \\
\hline Run, sprint & 27.60 & $28.67 \pm 0.52$ & 0.02 to 1.94 & 3.87 \\
\hline \multicolumn{5}{|c|}{ Nonlinear Indoor } \\
\hline Walk, jog & 27.60 & $28.55 \pm 0.83$ & -0.73 to 2.56 & 3.44 \\
\hline Run, sprint & 27.60 & $28.15 \pm 0.55$ & -0.39 to 1.65 & 1.99 \\
\hline
\end{tabular}


al. study, an issue that will need to be verified in future research.

The cumulative density function of the separation error is shown in Fig. 4. There is a close agreement between the linear and nonlinear trajectories in both the indoor and outdoor cases. In the outdoor experiment, the 90thpercentile separation error for both the linear and nonlinear courses is only $14 \mathrm{~cm}$, which is close to the absolute position error observed in the static measurement case. This result indicates that the relative position error outdoors is dominated by the absolute position errors of the individual nodes, and that there is no bias error. On the other hand, at the 90th percentile, the relative position error indoors is $35 \mathrm{~cm}$ for the linear course and $24 \mathrm{~cm}$ for the nonlinear course. These errors are slightly higher than the indoor absolute positional error, indicating the presence of bias error, which is to be expected due to the strong multipath conditions associated with indoor venues. The reason that the relative position error of the linear course is greater than that of the nonlinear course is due to the variation in the geometric dilution of precision. In particular, the nonlinear course was marked in the middle of the court, and thus enjoyed a better geometry, whereas the linear course ran from one end of the court to the other (refer to Fig. 1). The start and end segments of the linear course were near the court edges, resulting in a lower geometric dilution of precision. Another reason for the higher error observed in the linear course is that the mobile nodes were on the back of each athlete, so during the entire experiment period the anchor nodes on the front side were blocked by the body. In the nonlinear course, however, frequent direction changes allowed the mobile nodes to be in line of sight to different anchor nodes during the motion.

The sample estimated nonlinear course (red line) shown in Fig. 3 closely follows the nominal course (black dashed line). Although the participants were requested to travel along the nominal course as much as possible, due to the varying gait patterns of the participants, the actual course taken was not known. This potential experimental limitation must be considered in interpreting the results.

The error performance of the LPS distance estimation is shown in Table 1. Distance estimates are typically more accurate outdoors than indoors. For the linear course, the mean distance errors are $1.3 \%-1.4 \%$ outdoors and $2.0 \%-$ $2.4 \%$ indoors of the total distance travelled. The reason for this is that, as we have shown, range is estimated more accurately outdoors. The distance error and the standard deviation of the distance are higher for the nonlinear courses than for the linear courses. The highest mean errors are $2.4 \%$ for the linear course and $3.9 \%$ for the nonlinear course. Part of the reason for this is not increased measurement error, but the increase in an athlete's path variability, as it is much simpler to travel at speed in a straight line than to exactly follow a marked path on the ground. For the linear course in both venues, the distance was always overestimated by the system, which is evident from the fact that in both of these cases the minimum error is positive. The maximum error observed was $1.11 \mathrm{~m}$ for the indoor linear course, which is only $4 \%$ of the distance travelled. The mean error for the nonlinear course is higher in both venues than that for the linear course. The LPS underestimated the distance in certain experiments, as observed from the negative error values (however, it was possible to get from one end of the nonlinear course to the other while travelling less than the total distance by cutting corners), and the maximum error was $2.56 \mathrm{~m}$, which occurred in the indoor venue.

Our outdoor distance estimation results are comparable, in terms of mean and standard deviation, to the values reported in Frencken et al. (2010); however, in that study, distance was underestimated except on the linear course, which is contrary our results, in which all mean distances were overestimated. One reason for the difference in this observation is that the nonlinear courses used in the two studies were different. Another important reason is the amount of filtering that was applied in generating the trajectories. Unlike the LPS used in the study of Frencken et al., which outputs the location directly, the LPS we used provides ranges, and we used the algorithm presented in Hedley et al. (2011) to generate the positions of the players. As a result, we were able to vary the parameters of the filters used to study the effect of such filtering. What we observed was that for the nonlinear course, applying heavy filtering on the estimated locations resulted in a smoother track of the players. This did, however, cut the corners of the sharp turns, leading to significant underestimation of the distance travelled. When less (or no) filtering was applied, the variability of the tracks generated was higher, and the distance travelled was overestimated significantly. Therefore, the choice of the filtering algorithm for location tracking can affect the estimated distance, which has not been considered as a possible cause for the over- or underestimation of the distance in previous publications.

\section{Conclusions}

We presented the results of our study validating a radiofrequency LPS for tracking players in both indoor and outdoor sports. The results of our experiments showed that an appropriately designed radio-frequency LPS is a valid tool for performance analysis in sports and can provide consistent accuracy in both indoor and outdoor venues. Hence, sport scientists would benefit from utilising the data from an LPS to investigate current theories of group dynamics and how they relate to the actual performance of team sports (Araújo et al., 2006; Davids et al., 2008). Other 
benefits include the stability and variability of intra- and interpersonal coordination as a function of behaviour emerging from group action (Barris \& Button, 2008; Schmidt et al., 2011) and the creation of simulations of (sub)system behaviour that will model hypothesised game situations. Such a system would also provide valuable real-time information for sport scientists and coaches working with outdoor and indoor team sports on athlete positions, velocities, the relative distances and velocities between athletes, distances travelled, and spatial configurations on the field of play during critical events, such as transitioning from attack-defence-attack or penetrating a defensive structure. Finally, there is a need for future research to investigate the role of self-organisation processes in group learning tasks (Chow et al., 2007; Schmidt et al., 2011) and the role of constraints on self-organisation in learning (Newell, 1986), as well as to identify collective variables that can explain system behaviour at different levels of analysis.

Author note The authors thank their colleagues Alija Kajan (CSIRO) and Colin Mackintosh (AIS), for organising the field trials, and all of the volunteer participants.

\section{References}

Araújo, D., Davids, K., Chow, J.-Y., \& Passos, P. (2009). The development of decision making skill in sport: An ecological dynamics perspective. In D. Araújo, H. Ripoll, \& M. Raab (Eds.), Perspectives on cognition and action in sport (pp. 157-169). New York, NY: Nova Science.

Araújo, D., Davids, K., \& Hristovski, R. (2006). The ecological dynamics of decision making in sport. Psychology of Sport and Exercise, 7, 653-676.

Araújo, D., Davids, K., \& Serpa, S. (2005). An ecological approach to expertise effects in decision-making in a simulated sailing regatta. Psychology of Sport and Exercise, 6, 671-692.

Barris, S., \& Button, C. (2008). A review of vision-based motion analysis in sport. Sports Medicine, 38, 1025-1043.

Chow, J. Y., Davids, K., Button, C., Shuttleworth, R., Renshaw, I., \& Araújo, D. (2007). The role of nonlinear pedagogy in physical education. Review of Educational Research, 77, 251-278.
Davids, K., Button, C., \& Bennett, S. J. (2008). Dynamics of skill acquisition: A constraints-led perspective. Champaign, IL: Human Kinetics.

Duthie, G., Pyne, D., \& Hooper, S. (2003). The reliability of video based time motion analysis. Journal of Human Movement Studies, 44, 259-271.

Frencken, W. G. P., Lemmink, K. A. P. M., \& Delleman, N. J. (2010). Soccer-specific accuracy and validity of the local position measurement (LPM) system. Journal of Science and Medicine in Sport, 13, 641-645.

Glazier, P. S. (2010). Game, set and match? Substantive issues and future directions in performance analysis. Sports Medicine, 40, 625-634.

Gray, A. J., Jenkins, D., Andrews, M. H., Taaffe, D. R., \& Glover, M. L. (2010). Validity and reliability of GPS for measuring distance travelled in field-based team sports. Journal of Sports Sciences, 28, 1319-1325.

Hedley, M., Sathyan, T., \& Mackintosh, C. (2011). Improved wireless tracking for indoor sports. Proceedings of the 5th Asia-Pacific Congress on Sports Technology (pp. 439-444). Melbourne, Australia.

Hristovski, R., Davids, K., \& Araújo, D. (2009). Information for regulating action in sport: Metastability and emergence of tactical solutions under ecological constraints. In D. Araújo, H. Ripoll, \& M. Raab (Eds.), Perspectives on cognition and action in sport (pp. 43-57). New York, NY: Nova Science.

McGarry, T., Anderson, D., Wallace, S., Hughes, M., \& Franks, I. (2002). Sport competition as a dynamical self-organizing system. Journal of Sports Sciences, 20, 771-781.

Newell, K. M. (1986). Constraints on the development of coordination. In M. G. Wade \& H. T. A. Whiting (Eds.), Motor development in children: Aspects of coordination and control (pp. 341-360). Dordrecht, The Netherlands: Martinus Nijhoff.

Renshaw, I., Davids, K., Chow, J., \& Shuttleworth, R. (2009). Insights from ecological psychology and dynamical systems theory can underpin a philosophy of coaching. International Journal of Sport Psychology, 40, 580-602.

Sathyan, T., Humphrey, D., \& Hedley, M. (2011). WASP: A system and algorithms for accurate radio localization using low-cost hardware. IEEE Transactions on Systems, Man, and Cybernetics: Part C, 41, 211-222. doi:10.1109/TSMCC.2010.2051027

Schmidt, R. C., Fitzpatrick, P., Caron, R., \& Mergeche, J. (2011). Understanding social motor coordination. Human Movement Science, 30, 834-845.

Vilar, L., Araújo, D., Davids, K., \& Button, C. (2012). The role of ecological dynamics in analysing performance in team sports. Sports Medicine, 42, 1-10.

Zhu, J. (1992). Calculation of geometric dilution of precision. IEEE Transactions on Aerospace and Electronic Systems, 28, 893-895. 